\title{
Analyse organisationnelle et économique de la chaine de valeur du savon produit artisanalement à partir d'huile de Carapa procera DC. au Burkina Faso
}

\author{
Fanta Reine Sheirita Tiétiambou ${ }^{(1,2)}$, Anne Mette Lykke ${ }^{(3)}$, Urbain Dembélé ${ }^{(4)}$, \\ Abdelkader Aït El Mekki ${ }^{(5)}$, Gabin Korbéogo ${ }^{(6)}$, Amadé Ouédraogo ${ }^{(2)}$ \\ (1) Université Nazi BONI. Centre Universitaire de Gaoua. 01 BP 1091 Bobo-Dioulasso 01 (Burkina Faso). E-mail : \\ tietiambou.fanta@gmail.com \\ (2) Université Joseph Ki-Zerbo. Laboratoire de Biologie et Écologie Végétales. 03 BP 7021. Ouagadougou 03 (Burkina Faso). \\ (3) Aarhus University. Department of Bioscience. Vejlsøvej, 25. 8600 Silkeborg (Denmark). \\ (4) Institut d'Économie Rurale. Centre Régional de Recherche Agronomique de Sikasso. BP 186. Sikasso (Mali). \\ ${ }^{(5)}$ École Nationale d'Agriculture de Meknès. Département d'Économie Rurale. PO Box S/40. 50000 Meknès (Maroc). \\ (6) Université Joseph Ki-Zerbo. Département de Sociologie. 01 BP 1392. Ouagadougou 01 (Burkina Faso).
}

Reçu le 28 mars 2020, accepté le 23 juin 2020, mis en ligne le 7 septembre 2020.

Cet article est distribué suivant les termes et les conditions de la licence CC-BY (http://creativecommons.org/licenses/by/4.0/ deed.fr)

Description du sujet. Carapa procera DC. est un arbre oléagineux local à fort potentiel socio-économique. Sa promotion peut contribuer à diversifier et augmenter les sources de revenus des communautés locales.

Objectifs. Le but de cette étude est d'analyser la chaine de valeur (CV) du savon médical artisanal (SMA) de C. procera et d'évaluer son potentiel pour l'amélioration de l'économie locale. Notre analyse se limite aux dimensions organisationnelle et économique de cette $\mathrm{CV}$, notamment sur la création de valeur ajoutée et sa répartition entre les principaux acteurs qui la composent.

Méthode. Un inventaire des produits oléagineux de C. procera dans 29 marchés locaux et des enquêtes auprès de 104 acteurs ont été réalisés principalement dans le Kénédougou et dans d'autres localités de vente des produits au Burkina Faso.

Résultats. La CV du SMA implique au moins 283 acteurs réalisant 574 activités relatives à la collecte des graines, leur transformation primaire en huile, la transformation secondaire de l'huile en savon et la commercialisation du savon. Soixantetreize pour cent des acteurs sont membres d'une organisation et $71 \%$ des liens entre les acteurs sont basés sur des contrats. Les marges sont de $0,08 € \cdot \mathrm{kg}^{-1}$ de graines pour les collecteurs, $1,79 € \cdot \mathrm{l}^{-1} \mathrm{~d}$ 'huile pour les transformateurs primaires et respectivement 1,42 et $2,22 € \cdot \mathrm{kg}^{-1}$ de savon pour les transformateurs secondaires et les commerçants. Le revenu et la valeur ajoutée annuels perçus par l'ensemble des acteurs de la CV sont respectivement de $10573 €$ et $11335 €$. Le revenu total de la CV est inégalement distribué entre les acteurs, avec $20 \%$ d'entre eux qui en totalisent plus de $70 \%$.

Conclusions. L'essor de la CV du savon de C. procera nécessite une redistribution des revenus pour améliorer les parts des acteurs actuellement défavorisés.

Mots-clés. Coût, arbre fruitier, plante oléagineuse, revenu, traitement, Afrique de l'Ouest.

Organizational and economic analysis of the value chain for handmade soap from Carapa procera DC. oil in Burkina Faso

Description of the subject. Carapa procera is an oil tree that is widely grown in Burkina Faso. Its high socio-economic potential could contribute to the diversification and increase in sources of income for local communities.

Objectives. This study aimed to analyze the value chains of handmade medical soap made from C. procera seed oil and to assess potentials for improving the local economy. Our analysis is limited to organisational and economic dimensions from this value chains, in particular on the creation of added value and its distribution between the principal actors which make it up.

Method. An inventory of oil products from 29 local markets and interviews with 104 operators were conducted in Kénédougou province and other production localities in Burkina Faso. 
Results. The value chains of handmade medical soap included at least 283 operators with 574 activities including seed collection, primary transformation of seeds into oil, secondary transformation of oil into soap and trading of soap. Seventythree percent of operators were members of an organization and $71 \%$ of the relationships between operators were based on contracts. The margins were $0.08 € \cdot \mathrm{kg}^{-1}$ of seeds for seed collectors, $1.79 € \cdot \mathrm{l}^{-1}$ of oil for primary transformators, $1.42 €$. $\mathrm{kg}^{-1}$ and $2.22 € \cdot \mathrm{kg}^{-1}$ of soap for secondary transformators and traders, respectively. The total annual income perceived by all operators was $10,573 €$ and the total value added was $11,335 €$. The income was unequally distributed among the operators with $20 \%$ of them having more than $70 \%$ of the total income.

Conclusions. A redistribution of the income to improve the shares of the currently underprivileged operators is necessary in order to boost the value chains of handmade medical soap of C. procera.

Keywords. Costs, fruit trees, oil crops, income, processing, West Africa.

\section{INTRODUCTION}

Les écosystèmes forestiers ont un grand potentiel en produits forestiers non ligneux (PFNL) (Chominot, 2000 ; El Mansouri et al., 2011) dont l'exploitation procure des moyens de subsistance et des revenus aux populations locales des pays en développement (Shackleton \& Shackleton, 2004 ; Vodouhê et al., 2009). Au Burkina Faso, la production nationale de PFNL est estimée à 716004 tonnes en 2016 (DGESS, 2017). Une partie de cette production contribue à l'équilibre nutritionnel de plus de $43 \%$ des ménages ruraux, fournit $23 \%$ de revenus et procure 566353 emplois (DGEEVCC, 2018). L'autre partie, non négligeable, entre dans les échanges commerciaux internationaux du Burkina Faso. À titre d'exemple, entre $50 \%$ et $70 \%$ des noix brutes du karité exportées de l'Afrique de l'Ouest proviennent du Burkina Faso (Rousseau et al., 2015). La valeur ajoutée totale de ces PFNL commercialisés est de 271,85 milliards de francs CFA, représentant $3,85 \%$ du produit intérieur brut (PIB) national (DGEEVCC, 2018). La production nationale de PFNL reste dominée par les produits de cueillette non transformés constitués principalement des noix de karité (Vitellaria paradoxa C.F.Gaertn.), de la pulpe de fruits et de la poudre des feuilles de baobab (Adansonia digitata L.), des graines et pulpes de fruits de néré (Parkia biglobosa [Jacq.] G.Don), des graines $\mathrm{du}$ datier du Sahel (Balanites aegyptiaca [L.] Delile), des feuilles de tamarinier (Tamarindus indica L.), de la gomme arabique (Acacia senegal [L.] Willd.), des fruits de Saba senegalensis (A.DC.) Pichon, Lannea microcarpa A.Rich., Ziziphus mauritiana Lam. et de Acacia macrostachya DC. (APFNL, 2011). La commercialisation de produits de cueillette non transformés constitue une contrainte majeure à la création de valeur ajoutée et, par conséquent, un manque à gagner pour une économie locale et nationale.

$\mathrm{Au}$ regard des enjeux économiques qu'offre la transformation, la spécialisation vers des produits à haute valeur ajoutée est une option prometteuse. Dans ce sens, les graines oléagineuses de nombreuses plantes locales offrent des opportunités de valorisation
(Ouédraogo et al., 2013 ; Tiétiambou et al., 2016). Parmi ces plantes locales figure Carapa procera DC., un arbre local à graines oléagineuses dont le potentiel reste encore sous-utilisé. L'huile de cette plante a des propriétés médicinales (anti-inflammatoire, antitumorale), cosmétiques (soins des cheveux et de la peau) et biopesticides avérées (Konan et al., 2003 ; Djenontin et al., 2012 ; Nonviho et al., 2014 ; Dembélé et al., 2015). Cette huile sert à fabriquer une diversité de produits vendus sur les marchés (Guèye et al., 2010 ; Weber et al., 2010). Toutefois, ces divers produits n'ont pas les mêmes succès économiques. Il est donc nécessaire de cibler ceux qui sont porteurs, de les analyser pour identifier les facteurs qui vont favoriser la spécialisation et la professionnalisation des acteurs locaux dans une offre compétitive. C'est en ce sens que le savon médical artisanal (SMA) à base d'huile de graines de $C$. procera se présente comme un produit avec des potentialités de création de valeur ajoutée.

L'objectif général de l'étude est de déterminer la contribution socio-économique du savon médical artisanal à base d'huile des graines de C. procera à la réduction de la pauvreté au Burkina Faso. Les objectifs spécifiques sont :

- identifier les flux physiques des produits de la chaine de valeur (CV) du SMA;

- comprendre l'organisation interne des acteurs en termes de flux quantitatif et informatif autour de la production du savon ;

- estimer la rentabilité des produits intermédiaires de la CV ;

- évaluer des indicateurs de performance économique de la CV.

L'hypothèse qui sous-tend la présente étude est que le développement de la CV du SMA de C.procera peut contribuer à réduire la pauvreté au Burkina Faso, notamment dans les localités où la plante pousse naturellement. L'intérêt de cette étude est d'orienter dans l'identification des stratégies qui pourraient servir à promouvoir l'accès des petits producteurs au marché moderne. 


\section{MATÉRIEL ET MÉTHODES}

\subsection{Zone d'étude}

L'étude a été conduite principalement dans le Kénédougou, une province de la région des Hauts Bassins à l'ouest du Burkina Faso. La province a une population estimée à 370272 habitants en 2014, répartis entre quatre principales communautés que sont les Sénoufo, les Toussian, les Bolon et les Siamou. L'indice de pauvreté de la région des Hauts Bassins qui était de 34,4\% en 2014 est relativement faible par rapport au niveau global du pays $(40,1 \%$ en 2014) (INSD, 2014).

La provinde du Kénédougou bénéficie de conditions naturelles favorables au développement des activités agro-sylvo-pastorales du fait qu'elle fait partie des localités les plus arrosées du pays avec une pluviométrie moyenne estimée à $1008 \pm 164,7 \mathrm{~mm}$ par an entre 1983 et 2014. Les principales activités socioéconomiques sont l'agriculture (rente et subsistance) et l'élevage qui occupent plus de $95 \%$ de la population de la province. La cueillette des PFNL, qui est également très développée dans les villages, est surtout pratiquée par les femmes. L'étude a aussi concerné des localités de vente des produits oléagineux de $C$. procera. Il s'agit des provinces de la Comoé (Banfora), du Houet (Bobo Dioulasso) et du Kadiogo (Ouagadougou) (Figure 1).

\section{2. Échantillonnage et collecte des données}

L'étude a concerné un échantillon d'acteurs du réseau de production du SMA de C. procera. La méthode d'échantillonnage adoptée a été de type stratifié. Les

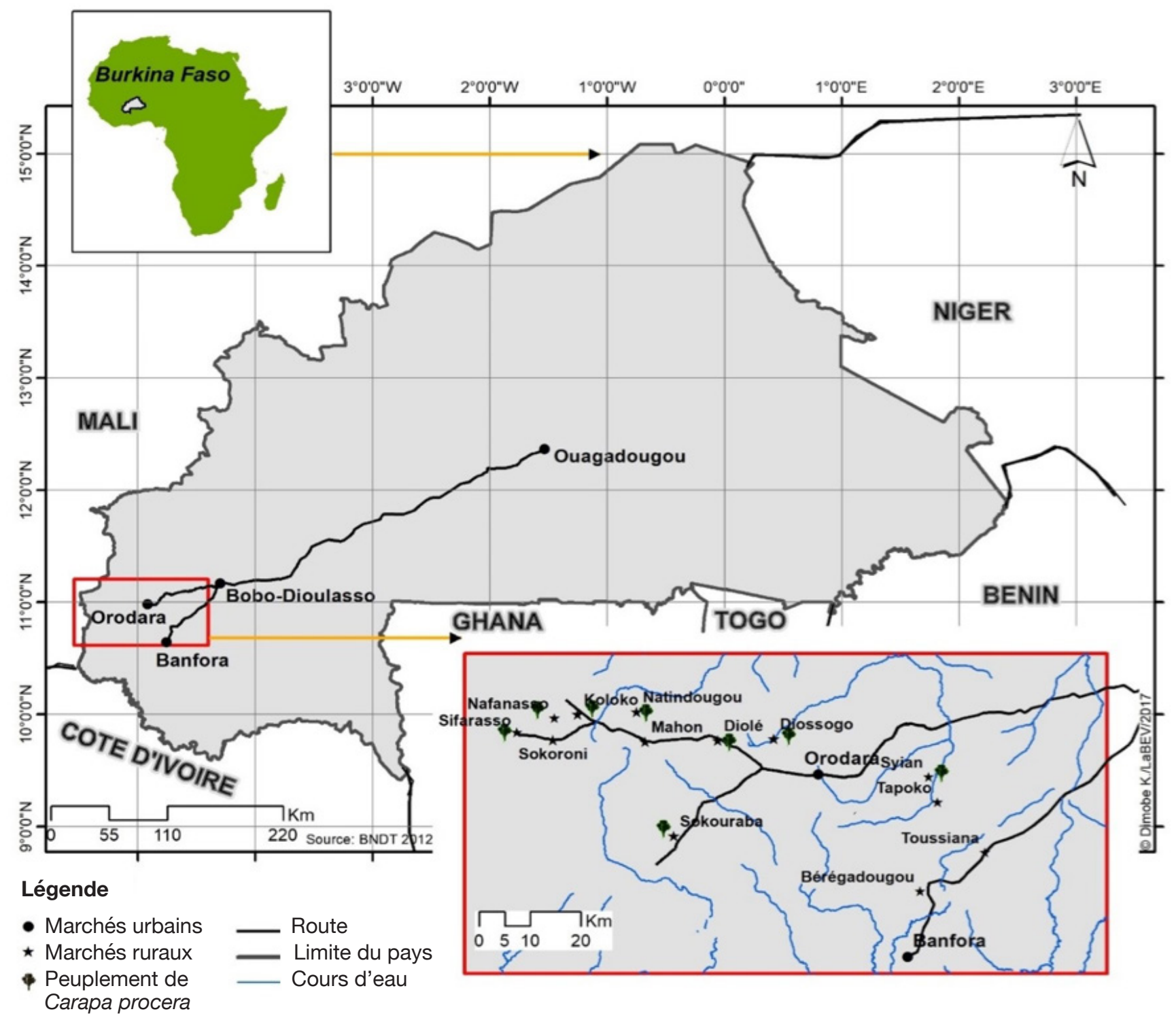

Figure 1. Sites d'étude - Study sites. 
strates sont représentées par les quatre maillons de la $\mathrm{CV}$ que sont la collecte des graines, la transformation primaire des graines en huile, la transformation secondaire de l'huile en savon et la commercialisation du savon. Dans chaque maillon, les acteurs ont été choisis en tenant compte de leur adhésion ou non à une organisation structurée et reconnue. Pour un niveau de précision acceptable des paramètres économiques évalués, des échantillons d'au moins 30 acteurs par maillon et indépendamment de l'étendue géographique ont été ciblés pour les enquêtes (Mbétid-Bessane, 2005). Dans les maillons ayant un nombre relativement faible d'acteurs, le maximum d'acteurs a été enquêté. L'identification des acteurs a été réalisée selon la méthode « boule de neige » (Wilhelm, 2014). Au total, 104 acteurs ont été enquêtés, répartis dans 11 marchés ruraux et 18 marchés urbains de quatre provinces (Tableau 1). Le questionnaire a été administré suivant des interviews structurées, concernant principalement les informations personnelles de l'acteur, son adhésion à une organisation d'acteurs, sa relation avec les autres acteurs (liens informels ou par contrats), ses quantités de produits échangés, ses prix de vente/achat et ses activités génératrices de revenus. Les données sur les valeurs monétaires ont été collectées en monnaie locale (franc CFA).

Aussi, l'ensemble des produits de la filière $C$. procera, à savoir les graines, 1 'huile et le savon ont été identifiés. Par la suite et pour chaque produit, ses zones de collecte, de transformation et de commercialisation ont été repérées.

\subsection{Analyse et traitement des données}

Flux quantitatif des acteurs et de leurs activités dans la production du savon. Un dénombrement de l'ensemble des acteurs au sein du réseau de production du SMAde la CV $\left(N_{o p}\right)$ a été réalisé. Le nombre d'acteurs membres d'une organisation a été obtenu en faisant la somme des individus membres des groupements du réseau. Le nombre d'acteurs individuels a représenté

Tableau 1. Nombre et répartition des acteurs par maillon de la chaine de valeur - Number and distribution of actors per link in the value chain.

\begin{tabular}{lll}
\hline $\begin{array}{l}\text { Maillon de la chaine de } \\
\text { valeur }\end{array}$ & $\begin{array}{l}\text { Acteur } \\
\text { individuel }\end{array}$ & Groupement \\
\hline Collecteurs de graines & 32 & 9 \\
Transformateurs primaires & 32 & 9 \\
$\begin{array}{l}\text { Transformateurs } \\
\text { secondaires }\end{array}$ & 10 & 1 \\
Commerçants de savon & 10 & 1 \\
\hline Total & $\mathbf{8 4}$ & $\mathbf{2 0}$ \\
\hline
\end{tabular}

la somme des individus exerçant seuls. Le nombre d'acteurs par maillon $\left(n_{o p}\right)$ est le nombre d'individus impliqués dans une même activité. Le nombre total d'activités représente l'ensemble des activités du réseau réalisé dans la $\mathrm{CV}$.

Flux informatif et organisation des acteurs dans la production du savon. L'analyse a consisté en une cartographie du réseau des acteurs de la production du savon (Faße et al., 2009). Pour cela, un ensemble de symboles standards de l'approche méthodologique value links (Springer-Heinze, 2007) a été adopté. Théoriquement, du fait que les acteurs se regroupent par affinité, leurs formes d'organisations et la nature des informations échangées entre eux déterminent la nature du pouvoir de gouvernance existant dans la chaine (Raikes et al., 2000 ; Gereffi et al., 2005). Ainsi, sur le plan vertical, le pourcentage de contrats entre acteurs a été déterminé. Sur le plan horizontal, le pourcentage d'adhésion des acteurs à une organisation formelle a été calculé.

Estimation des indicateurs de rentabilité financière de la CV. Le long de la CV, les séquences d'activités interdépendantes des acteurs ajoutent progressivement de la valeur à la matière première pour former le prix final du savon. Pour chaque produit, les paramètres suivants ont été calculés :

- le cout moyen de production unitaire $\left(C_{p}\right)$ suivant la formule de Springer-Heinze (2018) :

$$
C_{p}=\left[\sum_{1}^{n_{o p}}\left(\left(C_{f p}+C_{v p}\right) / Q_{p}\right) i\right] / n_{o p}
$$

avec $C_{f p}=$ les charges fixes de production; $C_{v p}=$ les charges variables de production ; $Q_{p}=$ le niveau de la production d'huile et de savon par individu ; $\left(C_{f p}+\right.$ $C_{v p} / Q_{p}=$ le cout total unitaire qui a été calculé pour chaque acteur direct, exprimé en $€ \cdot 1^{-1}$ pour l'huile et en $€ \cdot \mathrm{kg}^{-1}$ pour le savon.

- le cout moyen de commercialisation unitaire $\left(C_{c}\right)$ a été calculé similairement suivant la formule :

$$
C_{c}=\left[\sum_{1}^{n_{o p}}\left(\left(C_{f c}+C_{v c}\right) / Q_{c}\right) i\right] / n_{o p}
$$

avec $C_{f c}=$ les charges fixes de commercialisation ; $C_{v c}=$ les charges variables de commercialisation; $Q_{c}^{v c}=$ la quantité commercialisée de graines et de savon par individu ; $C_{c}$ est exprimé en $€ \cdot \mathrm{kg}^{-1}$.

Les activités de collecte de graines, d'extraction d'huile, de production de savon de $C$. procera et de commercialisation du savon ne constituent pas la principale activité chez les acteurs concernés. Le matériel utilisé se compose globalement des ustensiles de cuisine empruntés au ménage et du matériel utilisé pour d'autres productions. Pour ces raisons, les charges fixes sont négligeables et les 
couts se composent uniquement des charges variables de production (matière première, combustible, eau, additif $\mathrm{Na} / \mathrm{K}$ ) ou de commercialisation (carburant, frais de déplacement).

L'équation (1) devient :

$$
C_{p}=\left[\sum_{1}^{n_{o p}}\left(C_{v p} / Q_{p}\right) i\right] / n_{o p}
$$

L'équation (2) devient :

$$
C_{c}=\left[\sum_{1}^{n_{o p}}\left(C_{v c} / Q_{p}\right) i\right] / n_{o p}
$$

- la marge de production $\left(M_{p}\right)$ est établie selon la relation de Springer-Heinze (2018) :

$$
M_{p}=P_{v}-C_{p}
$$

Similairement, la marge de commercialisation $\left(M_{c}\right)$ a été calculée selon :

$$
M_{c}=P_{v}-C_{c}
$$

$P_{v}$ est le prix de vente unitaire du produit ; $M_{p}, M_{c}, P_{v}$, $C_{p}^{v}$ et $C_{c}$ sont exprimés en $€ \cdot \mathrm{kg}^{-1}$ pour les graines et le savon, et en $€ \cdot 1^{-1}$ pour l'huile. La marge de production ou de commercialisation représente le profit.

- le taux de rentabilité ( $r$ ) est obtenu par la formule :

$$
r=\frac{M}{P_{v}} \times 100
$$

- le revenu $(R)$ est estimé selon la formule :

$$
R=Q_{p} \times M
$$

$R$ est exprimé en $€, M$ représente la marge de production ou de commercialisation.

- la contribution du revenu à la réduction de la pauvreté monétaire $(C P)$ est établie selon la formule :

$$
C P=\frac{R}{S_{p}}
$$

$S_{p}$ représente le seuil de pauvreté de référence de l'année de collecte des données qui était de $234 €$ / personne adulte/an en 2014 (INSD, 2014).

Estimation des indicateurs de performance économique de la CV. La performance économique de la $\mathrm{CV}$ a été évaluée à travers le calcul des indicateurs suivants :

- l'emploi $(E)$ créé par la CV, par la formule :

$$
E=\left(N_{o p} \times T_{m}\right) / 8
$$

$T_{m}$ est le temps moyen (h) consacré à l'activité, ajusté des quantités produites. Huit heures de travail ont été considérées comme correspondant à une journée de travail (JT). E est exprimé en JT.

- le revenu d'un maillon est obtenu en multipliant le revenu perçu par chaque acteur direct par le nombre d'acteurs du maillon considéré. Ainsi, le revenu total de la CV a été obtenu en faisant la somme des revenus de l'ensemble des maillons.

- la valeur ajoutée créée par l'ensemble des acteurs de chaque maillon (VA) est estimée selon la formule suivante de Faße et al. (2009) :

$$
V A=C A-C I
$$

$C A$ représente le chiffre d'affaire en $€ ; C I$, la consommation intermédiaire en $€$.

La valeur ajoutée totale a été obtenue en faisant la somme des valeurs ajoutées de l'ensemble des maillons.

- l'indice d'inégalité de Gini $(G)$ est obtenu par la relation suivante de Lorenzo \& Paolo (2006) :

$$
G=1-\sum_{1}^{N_{o p}}\left[\left(q_{i}+q_{i-1}\right)\left(p_{i}-p_{i-1}\right)\right]
$$

$q_{i}$ est la proportion cumulée des revenus et $p_{i}$ est la proportion cumulée des acteurs.

Toutes les données ont été saisies dans le tableur Excel (C) et ont été examinées à travers les indicateurs de la statistique descriptive basés sur le calcul de la moyenne et des erreurs types. Les indicateurs qui concernent les valeurs monétaires ont été calculés en francs CFA avant d'être convertis et exprimés en $€(655,956$ francs $\mathrm{CFA}=1 €)$. Les analyses ont consisté d'abord à établir les relations entre les acteurs pour définir le type de gouvernance dans la CV. La détermination du type de gouvernance a été basée sur le système de coordination selon la classification de Gereffi et al. (2005). Puis les indicateurs de rentabilité financière ont été déterminés pour les acteurs de la CV. Enfin, ceux de la performance économique ont été utilisés pour une évaluation globale de la CV. La courbe de Lorentz des acteurs (Lorenzo \& Paolo, 2006) a été réalisée pour illustrer la distribution du revenu entre les acteurs.

\section{RÉSULTATS}

\subsection{Flux physique des produits et délimitation de la CV du savon}

Les graines de C.procera sont ramassées sous les arbres dans la forêt pour en extraire l'huile brute. Les zones de production de l'huile brute sont les villages des provinces du Kénédougou, de la Comoé et du 
Houet (Figure 2). L'huile produite dans ces villages est acheminée dans les chefs lieux des provinces (Banfora, Bobo Dioulasso et Orodara) où elle est transformée artisanalement en savon médical. Les zones de commercialisation du savon au Burkina Faso sont les régions de la boucle du Mouhoun, du centre et du sudouest. Dans la sous-région, le savon est commercialisé au Mali et en Côte d'Ivoire. La CV considérée dans cette étude porte sur la production du savon destinée uniquement au marché national.

\subsection{Flux quantitatif des acteurs et de leurs activités dans la production du savon}

Un total de 283 acteurs a été dénombré comme faisant partie du réseau organisé autour de la production du savon au Burkina Faso, dans les provinces du Kénédougou, de la Comoé et du Houet. Ces acteurs sont répartis dans quatre maillons correspondant aux activités de :

- collecte des graines de $C$. procera ;

- transformation primaire correspondant à l'extraction de l'huile des graines ;

- transformation secondaire portant sur la production du SMA à partir de l'huile ;

- commercialisation du SMA qui concerne l'achat et la vente du savon.

Seuls $2 \%$ des acteurs se retrouvent exclusivement dans le maillon de la commercialisation. La majorité des acteurs est répartie dans les autres maillons en assurant plusieurs activités à la fois. L'ensemble des acteurs a réalisé au total 574 activités dont $97 \%$ sont assurées par les transformatrices primaires qui interviennent également dans la collecte des graines (Figure 3).

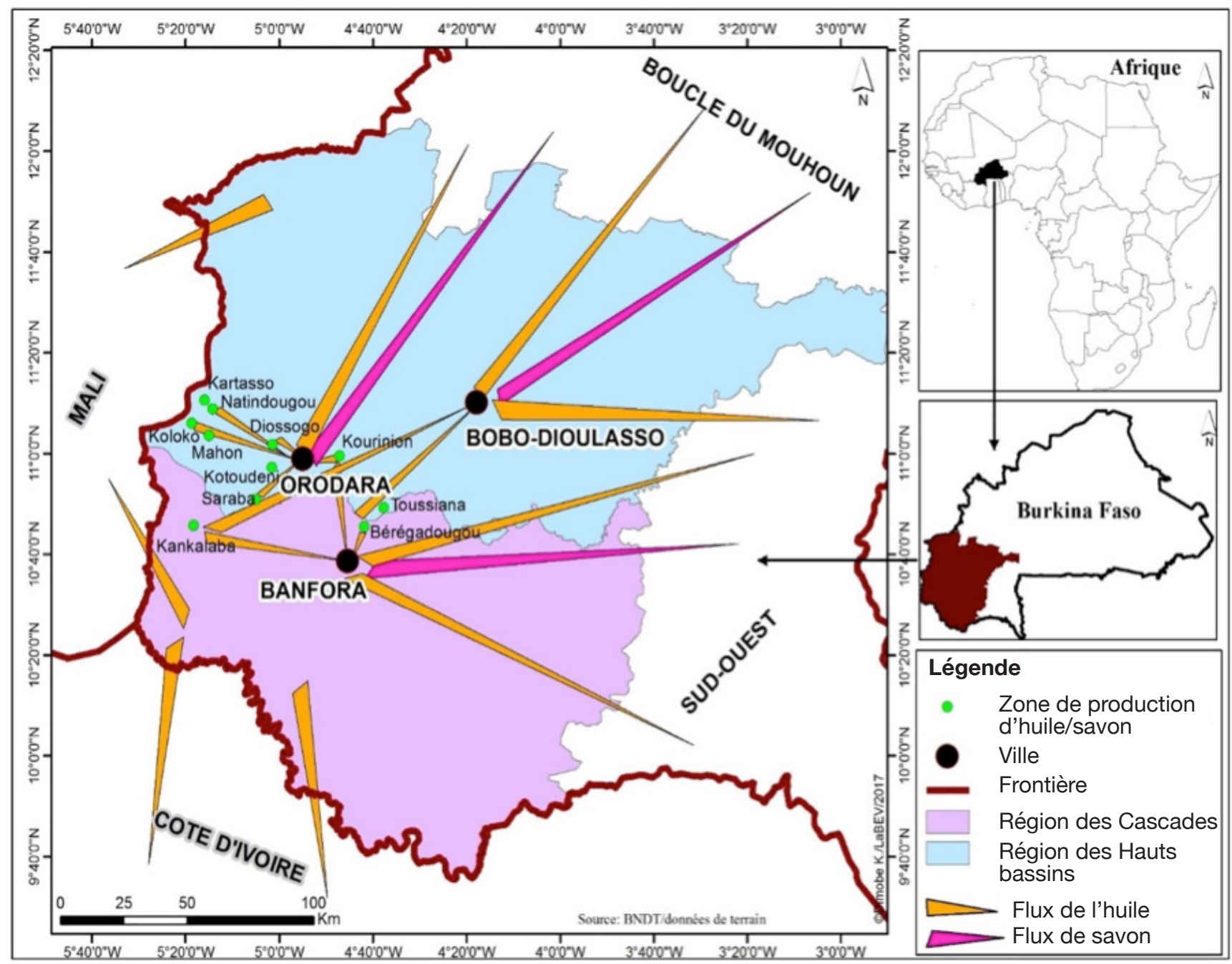

Figure 2. Cartographie des flux de l'huile et du savon de Carapa procera - Mapping of the flows of Carapa procera oil and soap. 


\subsection{Flux informatif et organisation des acteurs dans la production du savon}

Sur le plan horizontal, $27 \%$ des acteurs mènent individuellement leurs activités dans la CV, alors que les autres $(73 \%)$ le font dans le cadre de 14 groupements villageois. Une partie des acteurs des groupements $(62 \%)$ sont membres de deux unions et le reste des acteurs (11\%) sont regroupés dans une association (Figure 3). Le maillon de la transformation secondaire totalise la plus forte proportion d'acteurs adhérant à une organisation (100\%). Dans ce maillon,

\section{Nombre d'acteurs} et d'activités

\section{Acteurs du niveau micro-économique}

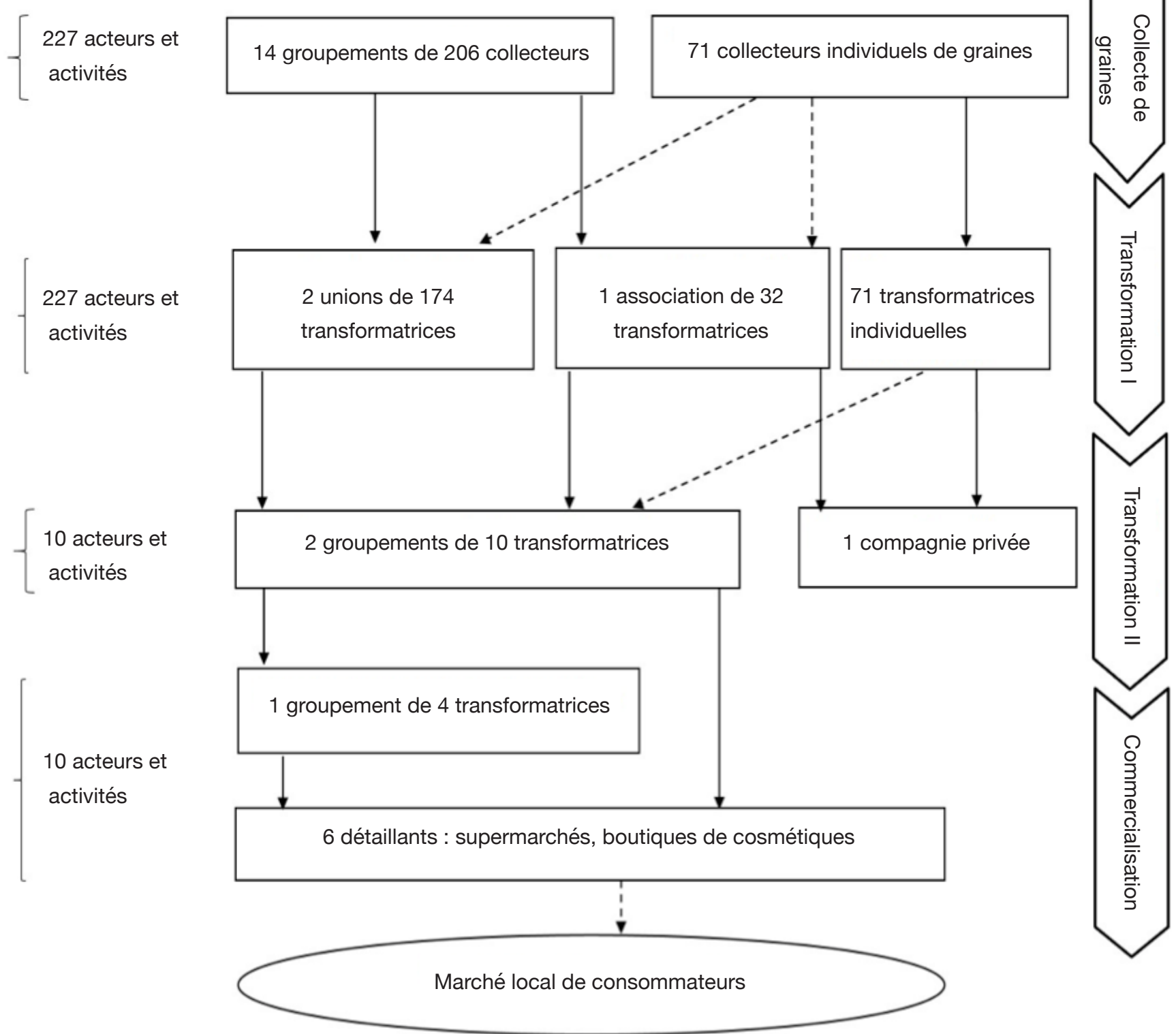

Figure 3. Cartographie des acteurs de la chaine de valeur du savon - Mapping of actors in the soap value chain.

Transformation I : transformation primaire - primary transformation ; Transformation II : transformation secondaire - secondary transformation; Symboles standards de l'approche chaine de valeur utilisée - Standard symbols of the value chain approach used : relation de lien informel entre acteurs - informal relationship between actors : $(\downarrow)$; relation contractuelle entre acteurs - contractual relationship between actors : $(\downarrow)$; marché - marketplace : (๖); acteurs - actors : $(\square)$; maillon de la chaine de valeur - link in the value chain: (\). 
la dynamique est à la pleine coordination des acteurs et les relations entre ces derniers concernent la soustraitance.

Sur le plan vertical, les acteurs communiquent à travers des liens informels $(29 \%)$ et des contrats (71\%). Les contrats sont plus fréquents au niveau des maillons de la transformation (primaire et secondaire) et de la commercialisation (> $70 \%$ ) (Tableau 2). Par contre, dans le cas de la collecte des graines, les acteurs individuels écoulent leurs produits aux groupements à travers des liens informels.

\subsection{Rentabilité financière des produits de la $\mathrm{CV}$ du savon}

La figure 4 donne la répartition des couts moyens de production supportés par les acteurs des maillons de la transformation primaire (a) et de la transformation secondaire (b). Pour l'ensemble des acteurs de ces

Tableau 2. Degré de coopération entre les acteurs de la chaine de valeur du savon médical artisanal - Degree of cooperation between the actors in the value chain of the handmade medical soap.

\begin{tabular}{lll}
\hline $\begin{array}{l}\text { Maillon de la } \\
\text { chaine de valeur }\end{array}$ & $\begin{array}{l}\text { Proportion } \\
\text { de contrats } \\
(\%)\end{array}$ & $\begin{array}{l}\text { Proportion } \\
\text { d'adhésion des } \\
\text { acteurs à une } \\
\text { organisation }(\%)\end{array}$ \\
\hline Collecte de graines & 60,00 & 74,37 \\
\hline $\begin{array}{l}\text { Transformation } \\
\text { primaire }\end{array}$ & 70,00 & 74,37 \\
\hline $\begin{array}{l}\text { Transformation } \\
\text { secondaire }\end{array}$ & 85,71 & 100,00 \\
\hline Commercialisation & 75,00 & 40,00 \\
\hline
\end{tabular}

a

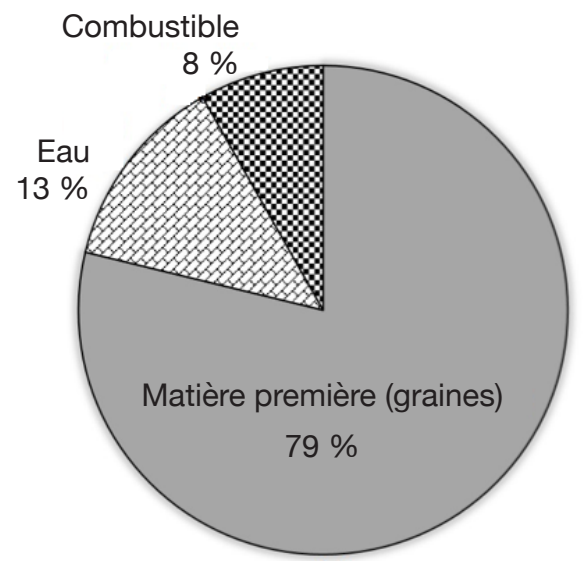

deux maillons, le cout de la matière première supportée représente plus de $75 \%$ des charges variables de production. Quant aux acteurs des maillons de la collecte des graines et de la commercialisation du savon, les charges variables de commercialisation sont négligeables. En conséquence, pour ces acteurs, nous pouvons considérer que les couts moyens de productions représentent $100 \%$ de la valeur du prix d'achat des graines et du savon. Ainsi, pour les acteurs du maillon de la collecte de graines, les couts moyens de commercialisation sont nuls.

En termes de valeurs, les couts moyens de production/commercialisation de l'ensemble des produits (graines, huile brute et savon) varient de 0 pour les acteurs de collecte des graines à $4,13 €$ pour ceux de la commercialisation du savon. Dans chaque maillon, toutes les charges variables supportées par les acteurs sont couvertes par le prix de vente du produit, ce qui donne des marges positives pour l'ensemble des produits. Aussi, les acteurs des maillons de la collecte des graines et de la transformation primaire réalisent des marges de plus de $50 \%$ du prix de vente par rapport aux autres acteurs des deux autres maillons qui en réalisent moins (Tableau 3).

Le tableau 4 présente, pour chaque maillon, la production par individu, le revenu généré par cette production individuelle et la contribution de ce revenu à la réduction de la pauvreté. Entre les acteurs, le revenu varie d'un montant d'environ $3 €$ perçus par acteur du maillon de la collecte des graines à $148 €$ perçus par acteur de la commercialisation. Pour ces acteurs, ces revenus représentent respectivement $1 \%$ et $63 \%$ du seuil de pauvreté au Burkina Faso. Les contributions à la réduction de la pauvreté sont plus élevées (> 50\%) pour les acteurs des maillons de la transformation secondaire et de la commercialisation.

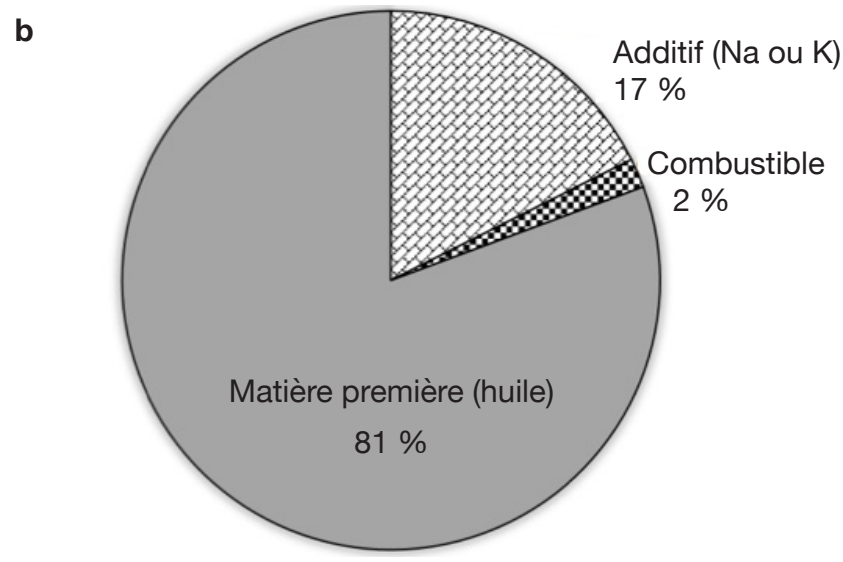

Figure 4. Répartition des couts supportés par les acteurs des maillons de la transformation primaire (a) et de la transformation secondaire (b) de la chaine de valeur du savon - Distribution of costs incurred by the actors in the primary (a) and secondary (b) transformations of the soap value chain. 
Tableau 3. Marges des acteurs et rentabilité des produits de la chaine de valeur du savon médical artisanal - Margins of the actors and profitability of the products of the artisanal medical soap value chain.

\begin{tabular}{llllc}
\hline Maillon de la CV & $\begin{array}{l}\text { Prix de vente moyen } \\
(€)\end{array}$ & $\begin{array}{l}\text { Cout moyen de production / } \\
\text { commercialisation }(€)\end{array}$ & $\begin{array}{l}\text { Marge } \\
(€)\end{array}$ & $\begin{array}{l}\text { Rentabilité } \\
(\%)\end{array}$ \\
\hline Collecte de graines (par kg) & $0,08 \pm 0,00$ & $0,00 \pm 0,00$ & 0,08 & 100,00 \\
Transformation primaire (par 1) & $2,10 \pm 0,07$ & $0,31 \pm 0,01$ & 1,79 & 85,44 \\
Transformation secondaire (par kg) & $4,13 \pm 0,16$ & $2,70 \pm 0,77$ & 1,43 & 34,42 \\
Commercialisation (par kg) & $6,35 \pm 0,00$ & $4,13 \pm 0,16$ & 2,22 & 35,00 \\
\hline
\end{tabular}

Tableau 4. Revenu et contribution à la réduction de la pauvreté des acteurs de la chaine de valeur du savon médical artisanal - Income and contribution to poverty reduction of actors in the artisanal medical soap value chain.

\begin{tabular}{llcc}
\hline $\begin{array}{l}\text { Maillon de la chaine de } \\
\text { valeur }\end{array}$ & $\begin{array}{l}\text { Quantités vendues / } \\
\text { opérateur }\end{array}$ & Revenu / acteur $(€)$ & $\begin{array}{l}\text { Contribution à la réduction de la pauvreté } \\
\text { monétaire }(\%)\end{array}$ \\
\hline Collecte de graines & $42,53 \pm 1,39 \mathrm{~kg}$ & 3,40 & 1,45 \\
Transformation primaire & $14,03 \pm 0,461$ & 25,11 & 10,73 \\
Transformation secondaire & $83,40 \pm 7,90 \mathrm{~kg}$ & 119,26 & 50,95 \\
Commercialisation & $66,72 \pm 8,42 \mathrm{~kg}$ & 148,12 & 63,29 \\
\hline
\end{tabular}

Tableau 5. Emplois créés et contribution de la chaine de valeur du savon médical artisanal au revenu et à la création de valeur ajoutée au niveau des acteurs - Jobs created and contribution of the artisanal medical soap value chain to income and added value creation at the actors level.

\begin{tabular}{lcccc}
\hline $\begin{array}{l}\text { Maillon de la chaine de } \\
\text { valeur }\end{array}$ & $\begin{array}{l}\text { Emploi créé } \\
\text { (journées de travail) }\end{array}$ & $\begin{array}{l}\text { Consommation } \\
\text { intermédiaire }(€)\end{array}$ & $\begin{array}{l}\text { Revenu des acteurs } \\
(€)\end{array}$ & $\begin{array}{l}\text { Valeur ajoutée par } \\
\text { les acteurs }(€)\end{array}$ \\
\hline Collecte de graines & 349,89 & 0 & 942,46 & 942,46 \\
\hline Transformation primaire & 3036,18 & 942,04 & 6956,49 & 7219,21 \\
\hline Transformation secondaire & 354,59 & 1751,40 & 1192,62 & 1693,02 \\
Commercialisation & - & 2755,54 & 1481,18 & 1481,18 \\
\hline Chaine de valeur du savon & 3740,66 & 5448,98 & 10572,76 & 11335,25 \\
\hline
\end{tabular}

\subsection{Performances économiques de la CV du savon}

En termes de potentialités d'emplois, la CV du savon destiné au marché national mobilise au minimum 3741 journées de travail (JT) (Tableau 5) pour la production de $834 \mathrm{~kg}$ de savon d'un poids moyen unitaire de $0,12 \pm 0,005 \mathrm{~kg}$; la transformation primaire occasionne plus d'emplois avec une contribution de 3036 JT consacrés à l'extraction annuelle d'huile. Seuls $80 \%$ de la production en savon sont destinés à la commercialisation sur le marché national. Le reste est autoconsommé par les acteurs dans leurs ménages. Globalement, le revenu et la valeur ajoutée actuels perçus par l'ensemble des acteurs sont respectivement de $10573 €$ et de $11335 €$.
La courbe de Lorenz de la distribution des revenus de l'ensemble des acteurs s'éloigne de la droite d'équidistribution, indiquant une tendance à la baisse de l'aire de concentration maximale (Figure 5). L'aire de concentration maximale représente la surface entre la courbe de Lorenz et l'axe des abscisses. Il ressort également que $80 \%$ des acteurs ont moins de $30 \%$ du revenu total de la CV. Aussi, l'indice de Gini prend la valeur 0,79 , confirmant l'existence d'une grande inégalité entre les acteurs pour la répartition des revenus dans la CV. Relativement à chaque maillon, cet indice est de 0,57 pour les transformatrices primaires, 0,54 pour les transformatrices secondaires et de 0,56 pour les commerçants. Les inégalités de revenus sont bien plus faibles au sein de chaque maillon que globalement. 


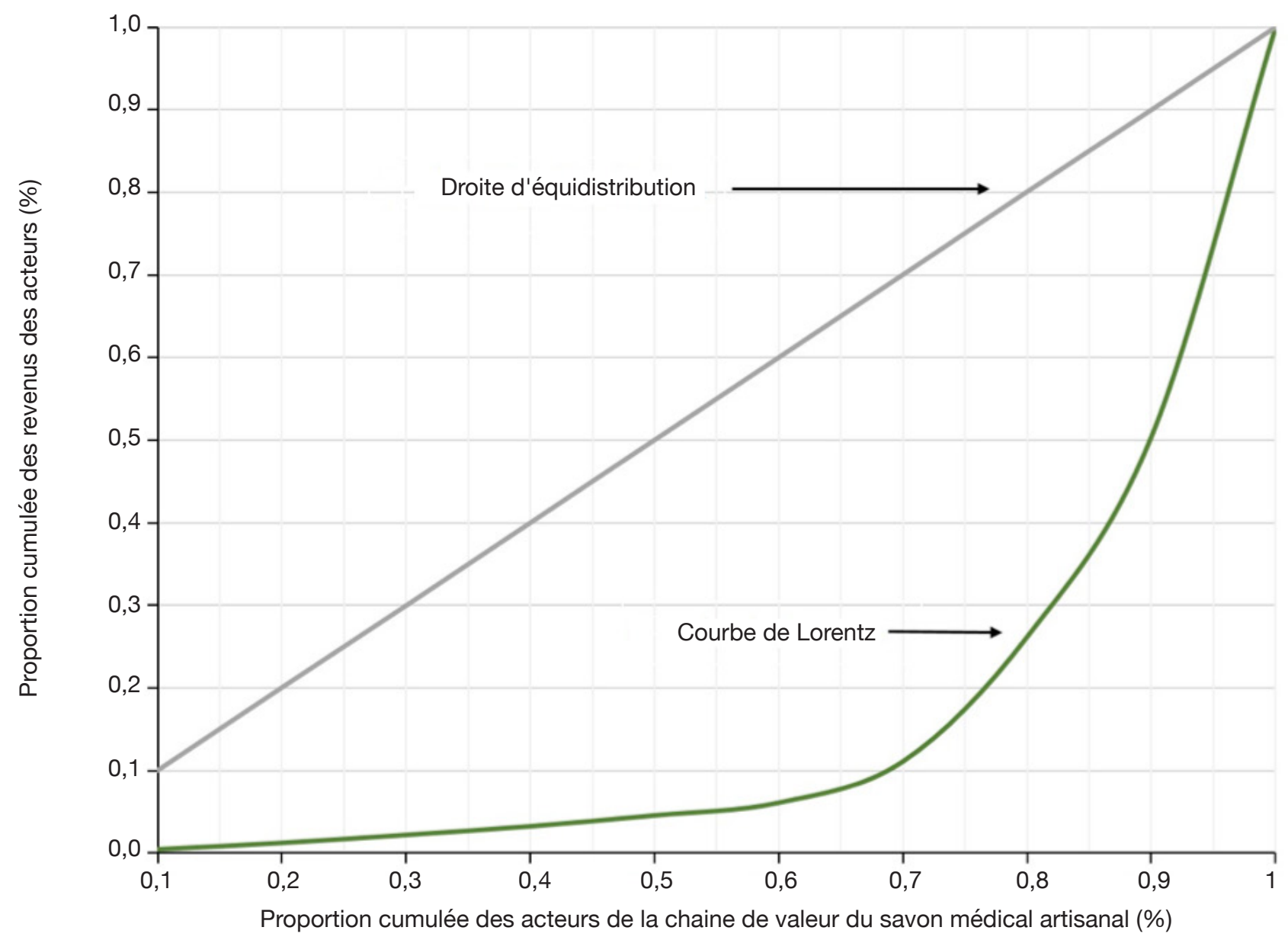

Figure 5. Distribution des revenus entre les acteurs de la chaine de valeur du savon - Distribution of income among actors in the soap value chain.

\section{DISCUSSION}

\subsection{L'organisation des acteurs et la gouvernance de la chaine de valeur ajoutée}

L'offre du savon sur le marché est assurée par quelques acteurs pour satisfaire la demande d'une multitude d'acheteurs. En effet, bien que le matériel de production du savon soit accessible à la plupart des acteurs, le savoir-faire de l'extraction d'huile n'est détenu que par quelques femmes d'un âge avancé (Tiétiambou et al., 2016). Selon la typologie de marché établie par Capul \& Garnier (2017), la structure d'un tel marché correspond au type oligopolistique. L'avantage de l'oligopole est que, du fait de leur nombre relativement faible, les offreurs vont investir davantage dans l'amélioration de leurs produits afin de pouvoir bénéficier d'un monopole temporaire. L'inconvénient est que les offreurs peuvent agir ensemble en collusion et avoir un contrôle sur les prix, peu importe la qualité du produit qu'ils offrent. Aussi, sur ce marché, le nombre d'acteurs (283) est deux fois inférieur au nombre d'activités qu'ils réalisent (574). Mis à part les acteurs du maillon de la commercialisation qui sont spécifiques à ce dernier, les autres acteurs mènent des activités diversifiées concernant différents maillons de la production du savon. Cela signifie qu'il n'y a pas de professionnalisation dans la répartition des activités de production du savon. L'avantage d'une professionnalisation est la garantie de la qualité par la valorisation du savoir-faire technique. Du point de vue de Coriat (1991), la maitrise du savoir-faire technique génèrerait des gains de productivité qui permettraient une réduction des couts dans la $\mathrm{CV}$ et favoriseraient son développement. Concernant la communication interne, les acteurs individuels écoulent leurs productions à travers des échanges aléatoires de marché. Selon la classification de Gereffi et al. (2005), la coordination est de type «marché ». Par contre, entre les acteurs d'un même maillon donné, il y a une communication structurée qui s'explique par le nombre relativement élevé d'individus intégrés dans des organisations. Les plus grandes organisations, constituées par les deux unions et l'association, sont les principaux acteurs dans 
la CV. Elles ont une grande influence sur la CV et sont par conséquent qualifiées de « parties prenantes » selon Freeman et al. (2010). Ces parties prenantes collectent l'huile produite par les groupements villageois de femmes transformatrices assurant la sous-traitance et l'avantage sur le long terme est d'apporter une certitude aux groupements villageois d'écouler leur marchandise (Bellon \& Fekih-Soussi, 2004 ; Courrent, 2012). Cependant, la durabilité d'un tel partenariat exige une répartition équitable des gains issus de cette relation (Baudry, 1993). Cette répartition équitable n'est pas constatée dans la CV du savon à base de l'huile des graines de C. procera. Actuellement, le revenu est inégalement distribué entre les acteurs et certains d'entre eux, qui sont défavorisés, pourraient sur le long terme quitter la chaine. Aussi, du fait que les parties prenantes sont guidées par leurs membres fournisseurs d'huile brute, la forme de gouvernance globale correspondante à la $\mathrm{CV}$ est de type captif. À leur tour, les parties prenantes fournissent une partie de leur huile brute à une compagnie privée. Aussi, du fait de l'absence de communication entre les parties prenantes, la compagnie privée a une position de force par le contrôle du prix à l'achat et les exigences de qualité de l'huile.

\subsection{La rentabilité financière et la performance économique de la CV}

Les produits de la CV du savon de C.procera ont permis à l'ensemble des acteurs de dégager des marges positives. On en déduit que chaque produit brut représenté par son prix de vente unitaire arrive à couvrir son cout de production/commercialisation. Les acteurs de la $\mathrm{CV}$ réalisent alors du profit. Il en résulte que les productions sont économiquement rentables (Paraïso et al., 2011). Dans le cadre de la collecte des noix de karité dans l'ouest du Burkina Faso, Rousseau et al. (2015) ont trouvé que la commercialisation de noix de karité occasionne une marge comprise entre 0,24 et $0,44 €$. Comparativement, la marge dégagée par la collecte des graines de $C$. procera est nettement inférieure. Cependant, la marge des acteurs de la commercialisation de noix de karité est distribuée entre six catégories d'acteurs au sein du même maillon, à savoir l'agriculteur/collecteur basé au village et fournisseur de noix, les intermédiaires du marché local, le détaillant provincial, le grossiste du marché régional, le détaillant du marché régional et les intermédiaires du marché régional. La plus grande marge est réalisée par le producteur/collecteur $(0,17$ à $0,30 €)$ et le reste est distribué entre les cinq autres intervenants. Par contre, dans le cas des activités de la transformation primaire et secondaire, la marge générée par l'extraction d'huile de $C$. procera est nettement supérieure. Cette marge est comprise entre 0,40 et $0,42 €$ par $\mathrm{kg}$ pour l'extraction du beurre de karité et entre 0,17 et 0,90 pour la production de produits dérivés du beurre (Badini et al., 2011). Aussi, les marges représentent plus de $50 \%$ du prix de vente de la production chez les acteurs de collecte des graines et de la transformation primaire de ces graines en huile, ce qui rend ces deux activités économiquement plus efficientes (Gharbi et al., 2007), leurs maillons dans la CV constituant une source d'avantages économiques pour la CV du savon.

Les marges positives permettent à l'ensemble des acteurs de la CV du SMA de réaliser des revenus positifs, contribuant ainsi à la réduction de la pauvreté monétaire dans la zone. On constate que, parmi les activités de la $\mathrm{CV}$, ce sont celles des maillons de la collecte des graines et de la transformation primaire des graines en huile qui contribuent le moins (inférieur à $15 \%$ ) à la réduction de la pauvreté par rapport aux autres qui contribuent à plus de $50 \%$. Ces maillons, qui comptent plus d'acteurs, justifient le déséquilibre constaté dans la distribution de revenus; ces maillons sont donc faibles. Malgré des performances intéressantes sur le plan organisationnel et économique, la $\mathrm{CV}$ du savon médical artisanal reste vulnérable.

\subsection{Des goulots d'étranglements fragilisent le développement de la $\mathbf{C V}$}

Certes, le savon est compétitif par son originalité, ses propriétés intéressantes (médicinale, cosmétique et insecticide) et par l'importance de la valeur ajoutée créée par rapport à la valeur de la production.

Toutefois, il a aussi des points faibles. Au niveau de ses caractéristiques physiques, le principal problème du savon est son caractère fondant rendant sa durée d'utilisation relativement courte. Cette faiblesse peut compromettre la compétitivité du savon en cas d'éventuelles entrées d'autres produits de substitution aux mêmes propriétés et à plus longue durée d'utilisation. Une innovation qui rendrait le savon plus dur est nécessaire pour renforcer sa compétitivité sur le marché et assurer la viabilité de la chaine. Au niveau du processus de création de la valeur, la qualité n'est pas garantie à cause du «savoir-faire technique » qui n'est pas toujours bien maitrisé par les acteurs. Comme l'a signifié Asanuma (1989), la qualité de la graine garantit la fabrication de produits de qualité. Dans la $\mathrm{CV}$ du savon, les savoir-faire techniques requis à la base pour que l'offre d'huile brute soit de qualité sont limités. Les huiles produites localement ne sont pas extraites suivant un standard, de sorte qu'elles sont de couleur variable (Dembélé et al., 2019), contiennent souvent des impuretés et ne sont pas conditionnées de façon appropriée. Les rendements de l'extraction de l'huile des graines de $C$. procera sont relativement faibles à cause des pertes de matière au cours du processus. Ce rendement d'extraction de l'huile par 
les transformatrices au Mali a été estimé à 23,1\% (Dembélé et al., 2019). Au niveau fonctionnel, les systèmes formels d'information sur les marchés sont faibles à cause de l'absence d'une bonne organisation des acteurs des différents maillons. Entre les acteurs de localités différentes, il y a une exclusion des acteurs individuels de la CV qui n'ont pas accès à l'information sur les prix de l'huile brute ni sur l'organisation interne existante. Cette situation crée des déséquilibres dans la fixation des prix dans le maillon de la transformation primaire et une sous-estimation de la valeur réelle des graines. Le prix des graines est bas, ce qui ne pousse pas les hommes à entrer dans le maillon de la collecte. Ces derniers s'orientent plutôt vers la commercialisation qui est financièrement plus rentable, comme cela est observé dans la filière karité avec le commerce des noix dont les prix de vente sont compris entre 0,17 et $0,30 € \cdot \mathrm{kg}^{-1}$ (Rousseau et al., 2015). Au niveau du marketing, la publicité générique reste locale. Seule la compagnie privée a développé un site web et des affiches publicitaires pour la promotion de son savon médical de C.procera. Toutefois, l'utilisation de l'huile dans la fabrication de savon médical artisanal peut être concurrencée selon Weber et al. (2010) par la demande accrue de l'utilisation de cette huile comme biopesticide naturel dans la culture du coton biologique. Cette situation peut affecter non seulement l'offre du savon médical artisanal dans la CV mais également le prix de l'huile brute et, par conséquent, le prix du savon dans la CV.

\subsection{Quelques points de leviers pour un meilleur choix stratégique de mise à niveau de la $\mathrm{CV}$}

La plus grande part du revenu de la CV du savon est absorbée par les commerçants et les fabricants de savons. Pourtant, ce sont les productrices d'huile qui constituent le maillon déterminant de la création de valeur ajoutée à travers la transformation des graines en huile. La recherche d'une approche d'équilibre parétienne où tous les acteurs sont satisfaits est alors nécessaire pour le développement et la durabilité de la CV. Une approche de mise à niveau dans le sens d'une redistribution de la valeur ajoutée est capitale. Cette mise à niveau qui peut concerner le produit, le processus de production ou les fonctions, améliore l'efficacité globale de la chaine de valeur (Gereffi et al., 2005). Le premier point de levier pour une mise à niveau fonctionnelle des acteurs de la CV est une meilleure organisation des acteurs à la base. Par exemple, un modèle de responsabilité sociale intégrée à la relation de sous-traitance dans une perspective « gagnant-gagnant » pourrait être bénéfique. En effet, ce modèle amplifie les effets économiques sur les acteurs et permet d'accroître leurs performances à long terme et le développement de la confiance entre partenaires (Courrent, 2012). Le deuxième point de levier devrait être orienté vers une stratégie qui vise l'amélioration des processus de transformation primaire et secondaire. Cela permettrait d'atténuer les pertes de matière au cours du processus, d'améliorer les rendements de production et de fournir des produits de qualité. Aussi, les acteurs devraient être amenés à uniformiser la production et acquérir une protection par marque collective pour ce savon. Ces acteurs peuvent tirer des avantages de cette protection s'ils produisent selon une norme de qualité et un standard pour offrir un produit homogène local typique de l'ouest du Burkina Faso. Devant le cout élevé de la protection, ces acteurs pourront bénéficier d'un accompagnement s'ils sont organisés en faitière ou en coopérative. Le troisième point de levier pourrait être axé sur l'adoption d'une stratégie de diversification des produits conventionnels dans des variantes de haute valeur "de-commodify » (Marescotti \& Belletti, 2016) où la fabrication de savon liquide serait un atout. Comme stratégie de de-commodify, les acteurs pourraient réduire la quantité d'huile de $C$. procera dans la production du savon tout en conservant ses propriétés médicinales et remplacer cette quantité par une huile moins couteuse, qui a des propriétés à la fois saponifiantes et cosmétiques. Il s'agit d'une substitution technique adoptée par les entreprises pour réduire le cout de revient d'un bien de production et acquérir un avantage concurrentiel. En définitive, la mise en place d' " alliances stratégiques » entre les producteurs du savon est impérative pour le développement durable de la filière C.procera et la CV du savon. Dans la chaine, la coordination doit être assurée par un groupe noyau. Cette forme d'organisation appelée «conscience écologique» par Guèye et al. (2010) facilitera la pénétration des productions locales sur le marché international.

\section{CONCLUSIONS}

L'analyse de la CV du savon a permis de juger de sa performance et d'apprécier son impact potentiel sur la réduction de la pauvreté au Burkina Faso. Bien que tous les acteurs contribuent à la chaine de fabrication du savon, les profits et l'accès à l'information sont caractérisés par une inégale répartition entre eux. Les plus grosses marges se retrouvent dans la collecte des graines et de la transformation primaire. Mais le paradoxe reste le faible niveau de revenu perçu par ces acteurs et le faible impact positif de ce revenu sur le seuil de pauvreté. Par ailleurs, le savon médical de $C$. procera présente une faiblesse dans ses caractéristiques physiques qui peut compromettre sa compétitivité face à d'autres savons et par conséquent la viabilité de sa chaine de valeur. Toutefois, sa production peut être optimisée par innovation et 
substitution technique. La vulgarisation de techniques améliorées de production du savon selon des normes de qualité auprès des transformatrices locales organisées en groupements formels pourrait apporter une contribution à l'amélioration de la qualité du savon. La quantité de savon produit pourrait alors augmenter considérablement pour satisfaire la demande et maximiser l'effet multiplicateur de la CV sur le revenu.

\section{Remerciements}

Les auteurs remercient les acteurs de la chaine de valeur du savon de l'huile de Carapa procera au Burkina Faso et la fondation DANIDA (Danish International Development Agency) qui a supporté ce travail à travers le projet de recherche QualiTree (Projet no.10-002AU).

\section{Bibliographie}

APFNL, 2011. Stratégie nationale de valorisation et de promotion des produits forestiers non ligneux. Burkina Faso: APFNL (Agence des Produits Forestiers Non Ligneux), MEDD (Ministère de l'Économie et du Développement Durable).

Asanuma B., 1989. Manufacturer-supplier relationships in Japan and the concept of relation-specific skill. J. Jpn. Int. Econ., 3, 1-30.

Badini Z., Kaboré M., Van der Mheen-Sluijer J. \& Vellema S., 2011. Chaînes de valeur de la filière karité au Burkina Faso. Wageningen, Pays-Bas : WUR.

Baudry B., 1993. Partenariat et sous-traitance : une approche par la théorie des incitations. Rev. Écon. Ind., 66, 51-68.

Bellon B. \& Fekih-Soussi B., 2004. Les effets des mutations de la sous-traitance sur les coûts de transaction, d'apprentissage et la croissance de l'entreprise. Rev. Région Dév., 20, 188-214.

Capul J.Y. \& Garnier O., 2017. Dictionnaire d'économie et de sciences sociales. Paris : Hatier.

Chominot A., 2000. Valorisation des plantes médicinales par l'industrie pharmaceutique, complémentarités et contradictions. Courrier Environ. INRA, 39, 19-26.

Coriat B., 1991. Penser à l'envers : travail et organisation dans l'entreprise japonnaise. Paris : C. Bourgois.

Courrent J.M., 2012. Développement durable et responsabilité sociétale en PME: comprendre pour agir. Bruxelles : De Boeck Supérieur.

Dembélé U. et al., 2015. Use-value and importance of socio-cultural knowledge on Carapa procera trees in the Sudanian zone in Mali. J. Ethnobiol. Ethnomed., 11, 14, doi.org/10.1186/1746-4269-11-14

Dembélé U. et al., 2019. Local perceptions and traditional methods for Carapa procera oil production in Mali. Flora Vegetatio Sudano-Sambesica, 22, 16-22, doi : 10.21248/fvss.22.77.
DGEEVCC, 2018. Stratégie nationale de développement de l'accès des produits forestiers non ligneux (PFNL) aux marchés. Burkina Faso: DGEEVCC (Direction Générale de l'Environnement de l'Économie Verte et du Changement Climatique), MEEVCC (Ministère de l'Environnement, de l'Économie Verte et du Changement Climatique).

DGESS, 2017. Annuaire des statistiques de l'environnement 2016. Burkina Faso : DGESS (Direction Générale des Études et des Statistiques Sectorielles), MEEVCC (Ministère de l'Environnement, de l'Économie Verte et du Changement Climatique) et PASF (Programme d'Appui au Secteur Forestier).

Djenontin T.S. et al., 2012. Composition of Azadirachta indica and Carapa procera (Meliaceae) seed oils and cakes obtained after oil extraction. Ind. Crops Products, 38, 39-45, doi.org/10.1016/j.indcrop.2012.01.005

El Mansouri L., Ennabili A. \& Bousta D., 2011. Socioeconomic interest and valorization of medicinal plants from the Rissani oasis (SE of Morocco). Bol. Latinoam. Caribe Plant. Med. Aromat., 10, 30-45.

Faße A., Grote U. \& Winter E., 2009. Value chain analysis methodologies in the context of environment and trade research, discussion papers. Hannover, Germany: Leibniz University.

Freeman R.E. et al., 2010. Stakeholder approach, the state of the art. Cambridge, UK: Cambridge University Press.

Gereffi G., Humphrey J. \& Sturgeon T., 2005. The governance of global value chains. Rev. Int. Pol. Econ., 12, 78-104.

Gharbi F.R., Lahsoumi R., Gouhis F. \& Rached Z., 2007. Rentabilité économique de l'élevage laitier en Tunisie : cas des Gouvernorats de l'Ariana et de Mahdia. Biotechnol. Agron. Soc. Environ., 11(3), 211-223.

Guèye M., Kenfack D. \& Forget P.M., 2010. Importance socioculturelle, potentialités économiques et thérapeutiques du Carapa (Meliaceae) au Sénégal. In: van der Burgt X., van der Maesen J. \& Onana J.M., eds. Systématique et conservation des plantes africaines. Kew, UK: Royal Botanical Gardens, 359-367.

INSD (Institut National de la Statistique et de la Démographie), 2014. Profil de pauvreté et d'inégalités. Ouagadougou, Burkina Faso : INSD (Institut National de la Statistique et de la Démographie), MEF (Ministère de l'Économie et des Finances).

Konan Y.L., Sylla M.S., Doannio J.M.C. \& Traoré S., 2003. Comparison of the effect of two excipients (karite nut butter and vaseline) on the efficacy of Cocos nucifera, Elaeis guineensis and Carapa procera oilbased repellents formulations against mosquitoes biting in Ivory Coast. Parasite, 10, 181-184.

Lorenzo G.B. \& Paolo L., 2006. Analyse d'inégalité : l'indice de Gini. Rome : FAO.

Marescotti A.\& Belletti G., 2016. Differentiation strategies in coffee global value chains through reference to 
territorial origin in Latin American countries. Cult. Hist. Digital J., 5(1), doi.org/10.3989/chdj.2016.007

Mbétid-Bessane E., 2005. Caractérisation du marché des huiles de karité en Centrafrique. Tropicultura, 23, 141145.

Nonviho G. et al., 2014. Chemical characterization of Lophira lanceolata and Carapa procera seed oils: analysis of fatty acids, sterols, tocopherols and tocotrienols. Res. J. Chem. Sci., 4, 57-62.

Ouédraogo A., Lykke A.M., Lankoandé B. \& Korbéogo G., 2013. Potentials for promoting oil products identified from traditional knowledge of native trees in Burkina Faso. Ethnobot. Res. Appl., 11, 071-083.

Paraïso A.A., Sossou A.C.G., Yegbemey R.N. \& Biaou G., 2011. Analyse de la rentabilité de la production du fonio (Digitaria exilis s.) dans la commune de Boukombe au Bénin. J. Rech. Sci. Univ. Lomé (Togo), 13(1), 27-37.

Raikes P., Friis Jensen M. \& Ponte S., 2000. Global commodity chain analysis and the French filière approach: comparison and critique. Econ. Soc., 29, 390417, doi.org/10.1080/03085140050084589

Rousseau K., Gautier D. \& Wardell A., 2015. Coping with the upheavals of globalization in the shea value chain: the maintenance and relevance of upstream shea nut supply chain organization in Western Burkina Faso. World Dev., 66, 413-427, doi.org/10.1016/j.worlddev.2014.09.004

Shackleton C. \& Shackleton S., 2004. The importance of non-timber forest products in rural livelihood security and as safety nets: a review of evidence from South Africa. S. Afr. J.Sci., 100, 658-664.
Springer-Heinze A., 2007. Manuel de value links : la méthodologie de la promotion de la chaîne de valeur. $1^{\mathrm{e}}$ éd. Eschborn, Allemagne: GIZ (Gesellschaft für Internationale Zusammenarbeit).

Springer-Heinze A., 2018. ValueLinks 2.0.: manual on sustainable value chain development. 2 vol. Eschborn, Germany: GIZ (Gesellschaft für Internationale Zusammenarbeit).

Tiétiambou F.R.S. et al., 2016. Perceptions et savoirs endogènes sur les espèces oléagineuses locales dans le Kénédougou (Burkina Faso). Bois For. Trop., 327, 3950, doi.org/10.19182/bft2016.327.a31295

Vodouhê F.G., Coulibaly O., Greene C. \& Sinsin B., 2009. Estimating the local value of non-timber forest products to Pendjari Biosphere Reserve dwellers in Benin. Econ. Bot., 63, 397-412, doi.org/10.1007/s12231-009-9102-7

Weber N. et al., 2010. L'huile de carapa (Carapa spp., Meliaceae) en Afrique de l'Ouest: utilisations et implications dans la conservation des peuplements naturels. Fruits, 65, 343-354, doi.org/10.1051/ fruits/2010029

Wilhelm M., 2014. Échantillonnage boule de neige, la méthode de sondage déterminé par les répondants. Rapport de méthodes. Neuchâtel, Suisse : Office Fédéral de la Statistique (OFS).

(37 réf.) 